\title{
Collisionless magnetic reconnection: analytical model and PIC simulation comparison
}

\author{
V. Semenov ${ }^{1}$, D. Korovinskiy ${ }^{1}$, A. Divin ${ }^{1}$, N. Erkaev $^{2,3}$, and H. Biernat ${ }^{4,5}$ \\ ${ }^{1}$ St. Petersburg State University, 198504, St. Petersburg, Russia \\ ${ }^{2}$ Institute of Computational Modelling, Russian Academy of Sciences, Siberian Branch, 660036, Krasnoyarsk, Russia \\ ${ }^{3}$ Siberian Federal University, 660041, Krasnoyarsk, Russia \\ ${ }^{4}$ Space Research Institute, Austrian Academy of Sciences, 8042, Graz, Austria \\ ${ }^{5}$ Institute of Physics, University of Graz, 8010, Graz, Austria
}

Received: 20 August 2008 - Revised: 26 January 2009 - Accepted: 26 January 2009 - Published: 2 March 2009

\begin{abstract}
Magnetic reconnection is believed to be responsible for various explosive processes in the space plasma including magnetospheric substorms. The Hall effect is proved to play a key role in the reconnection process. An analytical model of steady-state magnetic reconnection in a collisionless incompressible plasma is developed using the electron Hall MHD approximation. It is shown that the initial complicated system of equations may split into a system of independent equations, and the solution of the problem is based on the Grad-Shafranov equation for the magnetic potential. The results of the analytical study are further compared with a two-dimensional particle-in-cell simulation of reconnection. It is shown that both methods demonstrate a close agreement in the electron current and the magnetic and electric field structures obtained. The spatial scales of the acceleration region in the simulation and the analytical study are of the same order. Such features like particles trajectories and the in-plane electric field structure appear essentially similar in both models.
\end{abstract}

Keywords. Space plasma physics (Kinetic and MHD theory; Magnetic reconnection)

\section{Fast reconnection}

In 1964, Petschek presented a model of fast magnetic reconnection (Petschek, 1964), in which standing slow shock waves supply the conversion of magnetic energy. This mechanism, being much faster than the Sweet-Parker scheme (Sweet, 1958; Parker, 1957), explained the observed energy

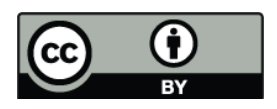

Correspondence to: V. Semenov (sem@geo.phys.spbu.ru) release rate. It was shown later that spatial non-uniform resistivity is required to support the Petschek type scenario (e.g. Sato and Hayashi, 1979; Biskamp, 1986; Erkaev et al., 2000; Biskamp and Schwarz, 2001; Erkaev et al., 2002). This anomalous resistivity in collisionless plasmas may be caused by microinstabilities, such as the lower hybrid drift instability (Huba et al., 1977), the Buneman instability (Drake et al., 2003), the ion-acoustic instability (Kan, 1971; Coroniti, 1985), or others (Büchner and Daughton, 2007).

However, another mechanism is developed using MHD with the Hall effect invoking (Sonnerup, 1979; Terasawa, 1983; Hassam, 1984), which causes a Petschek-like configuration due to the generation of dispersive waves (Mandt et al., 1994; Rogers et al., 2001) and does not require anomalous resistivity. It turns out that the contribution of the Hall effect appears close to the $X$-line, at length scales in order of the proton inertial length (skin depth), which is defined as $l_{p}=c / \omega_{p}$, where $c$ is the speed of light, $\omega_{p}=\sqrt{4 \pi n e^{2} / m_{p}}$ is the proton plasma frequency, and $m_{p}$ is the proton mass. Inside this region, the Hall effect decouples the proton(ion) and electron motions, tears protons off from the magnetic field lines, while the magnetic field remains frozen into the electron fluid.

Electrons demagnetize much closer to the $X$-line, inside the so-called electron diffusion region (EDR), because of inertia or non-gyrotropic pressure (Vasyliunas, 1975). A scaling analysis estimates $\delta \sim l_{e}$, where $\delta$ is the thickness of the $\mathrm{EDR}, l_{e}=c / \omega_{e}$ is the electron inertial length (skin depth), and $\omega_{e}=\sqrt{4 \pi n e^{2} / m_{e}}$ is the electron plasma frequency. Studies within the frame of the Geospace Environment Modeling (GEM) Magnetic Reconnection Challenge project (Birn et al., 2001) have shown that the Hall reconnection rate is insensitive to the dissipation mechanism activated in the EDR. The rate of magnetic reconnection is nearly independent of

Published by Copernicus Publications on behalf of the European Geosciences Union. 
the strength of dissipation in hybrid simulations with resistivity (Mandt et al., 1994), two-fluid simulations (Ma and Bhattacharjee, 1996; Biskamp et al., 1997), and particle-incell (PIC) simulations (Shay and Drake, 1998; Hesse et al., 1999). In addition, the Hall reconnection rate is independent of the magnitude of the electron mass (Shay and Drake, 1998; Hesse et al., 1999; Pritchett, 2001; Ricci et al., 2002) and the system size (Shay et al., 1999; Huba and Rudakov, 2004). The dependence on the electron dissipation and the system size seems to be found in studies of forced reconnection and double tearing mode reconnection (Grasso et al., 1999; Wang et al., 2001; Porcelli et al., 2002; Bhattacharjee et al., 2005). Summarizing, the results obtained by all types of numerical simulations, the essential feature of fast reconnection is the presence of the Hall effect, which provides the rate of the steady-state reconnection to be approximately a constant of the order of 0.1 .

\section{Analytical study}

Analytical studies of magnetic reconnection in the frame of Hall MHD (HMHD) meet difficulties that arise due to the EDR physics. However, as far as the reconnection rate does not depend on the mechanism of dissipation, one can restrict EDR contribution studies to the size of the EDR rather than its internal structure. Numerical simulations (Shay et al., 1998) confirmed the thickness of the EDR to be of the order of $l_{e}$ for anti-parallel Hall reconnection. The length of the EDR was thought to be $\sim 10 l_{e}$ (Shay et al., 1999; Huba and Rudakov, 2004), but later results suggested that it expands to the edge of the proton dissipation region, i.e., $\sim 10 l_{p}$ (Daughton et al., 2006). This contradiction seems to be resolved in recent PIC simulations which indicate that the EDR develops into a two-scale structure along the outflow direction. The length of the electron current layer is found to be sensitive to the proton/electron mass ratio, approaching $0.6 l_{p}$ for a realistic electron mass value. In addition, an elongated outflow electron jet is formed in the outflow region, and its length extends to $10^{\prime} \mathrm{s}$ of the proton skin depth (Shay et al., 2007).

Analytical studies of the problem may be simplified by using the electron Hall MHD (EHMHD) approximation. In the nearest vicinity of the stagnation point, at a length scale of the order of $l_{p}$, the proton velocities are small compared to the electron velocities. Hence, one may consider the electric current in this region as the electron current only (Biskamp, 2000),

$\mathbf{j} \approx-n e \mathbf{V}_{e}$

where $\mathbf{V}_{e}$ is the electron bulk velocity. For a detailed EHMHD-analysis of the problem, see Uzdensky and Kulsrud (2006). These authors have shown that for quasistationarity and translational symmetry assumed, the magnetic field and electron velocity can be expressed in terms of just a single one-dimensional function. This function is a magnetic potential of the in-plane magnetic field (poloidal flux function). In addition, the authors have found out that, neglecting the ion current, one gets the Grad-Shafranov equation for this potential.

In the work of Korovinskiy et al. (2008) an analytical model of self-consistent steady-state collisionless magnetic reconnection in an incompressible plasma was developed based on the Grad-Shafranov equation for the magnetic potential. We outline this model in next paragraphs and review the main results derived; in-depth study is provided in cited paper.

Firstly, we chose a coordinate system as follows: The Xaxis coincides with the magnetic field direction at infinity (in the upper semiplane), the Y-axis is directed along the $X$-line, and the $\mathrm{Z}$-axis is perpendicular to both of them. We assume homogeneity in the $Y$ direction, so all quantities are assumed to be independent of $Y$. We avoid the description of the EDR internal processes and consider only its size, which we suppose to be of the order of $l_{e}$ in its cross section ( $Z$ direction) and $\eta l_{p}$ along it ( $X$ direction), where $\eta$ is a coefficient of the order of 1. Outside the EDR the plasma is supposed to be nonresistive; furthermore, it is assumed to be quasi-neutral and incompressible.

The two-fluid description of our problem is determined by the following equations,

$$
\begin{aligned}
& \rho\left(\mathbf{V}_{p} \cdot \nabla\right) \mathbf{V}_{p}=-\nabla P_{p}+n e\left(\mathbf{E}+\frac{1}{c} \mathbf{V}_{p} \times \mathbf{B}\right), \\
& \mathbf{E}+\frac{1}{c} \mathbf{V}_{e} \times \mathbf{B}=-\frac{1}{n e} \nabla P_{e}, \\
& \nabla \times \mathbf{B}=\frac{4 \pi n e}{c}\left(\mathbf{V}_{p}-\mathbf{V}_{e}\right), \\
& \nabla \times \mathbf{E}=0, \\
& \nabla \cdot \mathbf{B}=0, \\
& \nabla \cdot \mathbf{V}_{p, e}=0 .
\end{aligned}
$$

Here, Eq. (2) is the equation of the proton motion, where $P_{p}$ is the scalar proton gas pressure, and $\mathbf{V}_{p}$ is the proton bulk velocity; Eq. (3) is the Ohm law, where $P_{e}$ is the scalar electron gas pressure; Eq. (4) is the Ampère law; Eq. (5) is the Faraday law; Eq. (6) is the Gauss law; and Eq. (7) is the mass conservation law for each particle species.

In our steady-state $2.5 \mathrm{D}$ case, the electric field $E_{y}$ must be a constant, according to Faraday's law (Eq. 5). So, we define

$E_{y}=\epsilon E_{A}$,

where $\epsilon$ is the reconnection rate which we assume to be small, $\epsilon \ll 1$, and $E_{A}=\frac{1}{c} B_{0} V_{A}$ is the Alfvén electric field. Here, $B_{0}$ is the magnetic field value above the $X$-line at the upper boundary of the examined region and $V_{A}$ is the corresponding proton Alfvén velocity.

To resolve the system (2-7), we introduce dimensionless quantities: The magnetic field strength $\tilde{\mathbf{B}}=\mathbf{B} / B_{0}$, the proton and electron bulk velocities $\tilde{\mathbf{V}}_{p, e}=\mathbf{V}_{p, e} / V_{A}$, the electric field 
strength $\tilde{\mathbf{E}}=\mathbf{E} / E_{A}$, the gas pressure $\tilde{P}_{p, e}=P_{p, e} / P_{0}$, and the length scales $\tilde{\mathbf{r}}=\mathbf{r} / l_{p}$. Here $P_{0}=B_{0}^{2} / 4 \pi$, and $\mathbf{r}=(x, y, z)$.

Secondly, we introduce an electric potential $\tilde{\Phi}$ via $\tilde{\mathbf{E}}=-\tilde{\nabla} \tilde{\Phi}$. Omitting the tildes, we rewrite Eqs. (2-7) for normalized quantities, bearing in mind the EHMHD approximation (1),

$\left(\mathbf{V}_{p} \cdot \nabla\right) \mathbf{V}_{p}=-\nabla P_{p}-\nabla \Phi$,

$\mathbf{V}_{e} \times \mathbf{B}=\nabla \Phi-\nabla P_{e}$,

$\nabla \times \mathbf{B}=-\mathbf{V}_{e}$,

$\nabla \cdot \mathbf{B}=0$,

$\nabla \cdot \mathbf{V}_{p, e}=0$.

Note that $\Phi$ has a linear dependence on $Y$ coordinate, so that $\partial \Phi / \partial y=-\epsilon$. Under this point of view, we can present $\Phi$ as a sum of two terms, namely $\Phi(x, y, z)=\phi(x, z)-\epsilon y$. Using an effective potential $\phi_{\mathrm{eff}} \equiv \phi-P_{e}$, we eliminate quantity $P_{e}$ from the Ohm law (10).

We also introduce a magnetic potential $A(x, z)$,

$\mathbf{B}_{\perp} \equiv\left(B_{x}, B_{z}\right)=\nabla \times\left(A \mathbf{e}_{y}\right)$,

where $\mathbf{e}_{y}$ is the unit vector and $\perp$ denotes the $X Z$ plane.

At last, we note that accordingly to the Ampère law (Eq. 11), the magnetic field $B_{y}$ is the stream function for the electron in-plane velocity (Biskamp, 2000),

$\mathbf{V}_{e \perp} \equiv\left(V_{e x}, V_{e z}\right)=-\nabla \times\left(B_{y} \mathbf{e}_{y}\right)$.

Bearing in mind the EHMHD approximation (1), we note that Eq. (10) looks very similar to the condition of magnetohydrostatic equilibrium,

$\nabla P=\mathbf{j} \times \mathbf{B}=\Delta A \mathbf{e}_{y} \times \mathbf{B}$.

In 2.5 D models, expression (16) yields the famous GradShafranov equation (Schindler, 2007),

$$
-\Delta_{\perp} A=\frac{d}{d A}\left(P(A)+\frac{1}{2} B_{y}^{2}(A)\right),
$$

where $\Delta_{\perp}$ is the $2 \mathrm{D}$ Laplace operator.

Analogously, we obtain

$V_{e y} \equiv \Delta_{\perp} A=\frac{d G(A)}{d A}$,

where $G(A)$ is an unknown modelling function. The other equations of the system (9-13) take the following form

$B_{y}(r)=(-1)^{k+1} \epsilon \int_{r_{0}}^{r} \frac{d s_{f l}}{\left|\nabla_{\perp} A\right|}+B_{y}\left(r_{0}\right)$,

$\phi_{\mathrm{eff}}=\frac{1}{2} B_{y}^{2}+G(A)$

$\frac{1}{2} V_{p \perp}^{2}+\Pi-\frac{1}{2}\left|\nabla_{\perp} A\right|^{2}+G(A)=C_{t r}$,

$\nabla_{\perp} \cdot \mathbf{V}_{p \perp}=0$

$V_{p y}(r)=\epsilon \int_{r_{0}}^{r} \frac{d s_{t r}}{V_{p \perp}}+V_{p y}\left(r_{0}\right)$.
Here, Eq. (19) is the equation for the out-of-plane magnetic field $B_{y}$, where $k$ is the quadrant number and $d s_{f l}$ is an elementary displacement along the projection of the magnetic field line onto the $X Z$ plane; Eq. (20) is the equation for the effective electric potential $\phi_{\text {eff }}$; Eq. (21) is the Bernoulli equation for the in-plane motion of protons, where $\Pi \equiv P_{p}+(1 / 2) B^{2}$ is a total pressure and $C_{t r}$ is a constant along the trajectory; Eq. (22) is the continuity equation, where $\mathbf{V}_{p \perp}$ is the proton in-plane velocity; and Eq. (23) is the equation for the out-of-plane proton velocity $V_{p y}$, where $d s_{t r}$ is an elementary displacement along the projection of the proton trajectory onto the $X Z$ plane.

Thus, the initial complicated system splits, and the solution of the problem bases on the solution of the GradShafranov equation for the magnetic potential (18). A scaling of the problem allows us to make use of the boundary layer approximation $\partial / \partial x \ll \partial / \partial z$. Under this approximation, the Laplace Eq. (18) has a following solution

$$
\begin{aligned}
z(A) & = \pm \frac{1}{\sqrt{2}} \int_{A_{0}}^{A} \frac{d A^{\prime}}{\sqrt{\left|G\left(A^{\prime}\right)-G\left(A_{0}\right)\right|}}, \\
A_{0} & \equiv A(x, 0)=\int_{0}^{x} B_{z}\left(x^{\prime}, 0\right) d x^{\prime},
\end{aligned}
$$

with the boundary condition $B_{z}(x, 0)$.

The unknown function $G(A)$ has a simple physical meaning, namely it is the main part of the electric potential, while its derivative is the out-of-plane electron velocity. This allows us to preset function $G(A)$ manually in order to obtain the solution of the problem (Korovinskiy et al., 2008), or get it from some other source, e.g., from PIC simulations.

Note that Eqs. (18-23) do not contain any dissipation so solution obtained is, strictly speaking, nonapplicable inside the EDR. Indeed, accordingly to Eq. (19) the magnetic field $B_{y}$ tends to infinity at the origin, where the in-plane magnetic field goes to zero. Therefore, we must interrupt the calculation of $B_{y}$ at the EDR boundary and have to consider this region separately. Advantageously, the EDR is very thin and comparatively short, the function $B_{y}(x, z)$ is smooth, and $B_{y}(0,0)=0$ due to the symmetry condition. Therefore, it is justified to neglect EDR contribution in $B_{y}$.

Equation (19) claims also that the extreme values of $B_{y}$ are located at the separatrices of the in-plane magnetic field, as well as extreme values of $|\nabla G(A)|$ and $\left|V_{e y}\right|$ are. Using the condition for solvability of Eq. (24), we obtain estimation of the extremum electric field, $\max |E| \sim 10 E_{A}$. Note that outside the EDR, the contribution of $\nabla P_{e}$ is negligible small, so $\phi \approx \phi_{\text {eff. }}$ As for the electron velocity $V_{e y}$, the Ampère law yields

$\max \left|V_{e y}\right|=\frac{1}{\delta} V_{A e}$,

where $\delta$ is the EDR width measured in electron skin depths $l_{e}$ and $V_{A e}=\sqrt{m_{p} / m_{e}} V_{A}$ is the electron Alfvén velocity. At last, the proton in-plane motion obeys the Bernoulli Eq. (21). 

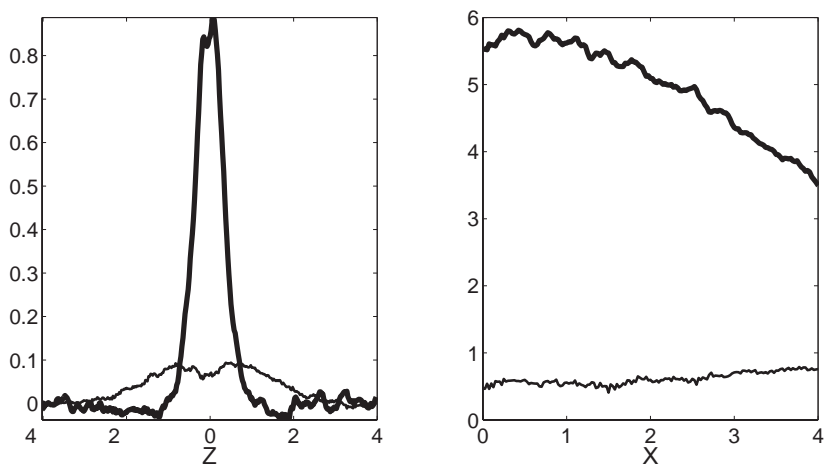

Fig. 1. PIC simulation data: Quantities $j_{e y}(0, z)$ thick and $j_{p y}(0, z)$ thin (on the left); quantities $\left|V_{e y}(x, 0)\right|$ thick and $V_{p y}(x, 0)$ thin (on the right).

Under the simple assumption $\Pi=$ const, the drop of the electric potential accelerates protons up to $V_{A}$. More realistically, the total pressure is fixed by its distribution at the upper boundary of the EHMHD domain, so that $\Pi=\Pi(x)$. Thus, the system of Eqs. (18-25) expresses a self-consistent solution of our problem based on the modelling function $G(A)$ with the boundary conditions $B_{z}(x, 0)$ and $\Pi\left(x, z_{\max }\right)$. To check the effectiveness of the model we take these functions from the PIC simulation of reconnection and then compare our analytical solution and the numerical model.

\section{PIC simulation}

The explicit particle-in-cell code P3D (Zeiler et al., 2002) is used for a simulation of $2.5 \mathrm{D}$ reconnection. In brief, the P3D is an electromagnetic full particle code; the Boris algorithm (Birdsall and Langdon, 1991) is used for the numerical solution of the equation of motion. The electromagnetic field solver uses leapfrog scheme to advance fields in time. For the initial condition, we take conventional a Harris neutral current sheet (Harris, 1962), where

$$
\begin{aligned}
B_{x} & =B_{0} \tanh \frac{z}{\lambda}, \\
n(z) & =n_{0} \cosh ^{-2}\left(\frac{z}{\lambda}\right)+n_{b},
\end{aligned}
$$

with a background plasma density $n_{b}=0.2$ and a half-width of the initial current sheet $\lambda=0.4 l_{p}$.

The magnetic field is normalized to its maximum value in the lobes and the density is normalized to its current sheet maximum. A moderate initial GEM-type perturbation (Birn et al., 2001) is added to ignite reconnection

$\Psi(x, z)=\Psi_{0} \cos \frac{2 \pi x}{L_{x}} \cos \frac{\pi z}{L_{z}}$,

where $L_{x}=L_{z}=38.4 l_{p}$ are the sizes of the computational box and the intensity of perturbation is $\Psi_{0}=0.3$.
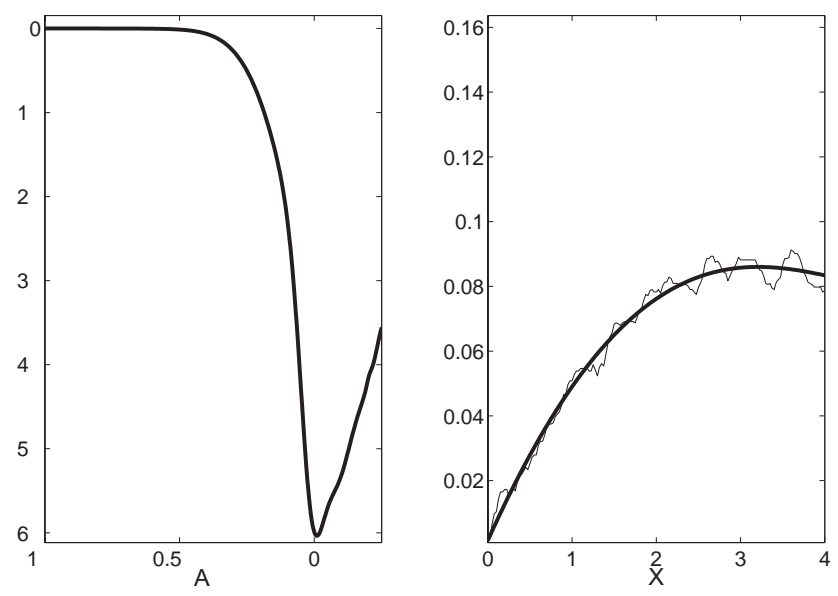

Fig. 2. PIC simulation data: Function $d G / d A \equiv V_{e y}(A)$ (on the left); magnetic field $B_{z}(x, 0)$ (on the right). The point $A=0$ on the left picture corresponds to the magnetic field separatrices. Simulation data are shown by the thin line, approximation is shown by the thick curve.

A quasistationary state is achieved at $t=15 \Omega_{p}^{-1}$, where $\Omega_{p}^{-1}$ is the inverse proton gyrofrequency (see Fig. 7), and the simulation parameters at $t=20$ are further taken as a reference to be compared with the analytical study. The mass ratio is $m_{p} / m_{e}=64$ and the temperature ratio is $T_{p} / T_{e}=3 / 2$.

Open boundary conditions for the fields

$\frac{\partial B_{x, y}}{\partial x}=0, \quad \frac{\partial E_{y}}{\partial x}=0, \quad E_{x, z}=0, \quad B_{z}=0$

and particles

$\frac{\partial n_{e, p}}{\partial x}=0, \quad \frac{\partial \mathbf{V}_{e, p}}{\partial x}=0, \quad \frac{\partial T_{e, p}}{\partial t}=0$

are implemented at the exhaust boundaries to allow a free outflow of the plasma (Divin et al., 2007; Pritchett, 2001).

A perfect electric conductor (PEC) boundary closes the simulation box at $z= \pm 19.2$. Under the boundary conditions adopted, not more than $15 \%$ of magnetic flux and particles escape through the outflow boundary by $t=20$. In the following section, the results of our simulations are presented.

\section{Comparison of the results}

A plot of the electric current $j_{e y}$ at $x=0$ is presented in Fig. 1 at the left, where the EDR is a well-recognizable region where the electron current dominates over the proton one. The EDR half-width $\delta$ comes up to $3 / 4 l_{p}$, i.e., $\delta \approx 6 l_{e}$ under the used mass ratio. According to our estimation (26), the analytical model gives $\max \left|V_{e y}\right| \approx 7 V_{A}$, and the value obtained in the PIC simulation is $6 V_{A}$ (see Fig. 1, right). The electron/proton current ratio is $j_{e} / j_{p} \approx 11$ in the origin and it decreases moving away from the $X$-line. As far as the EHMHD 

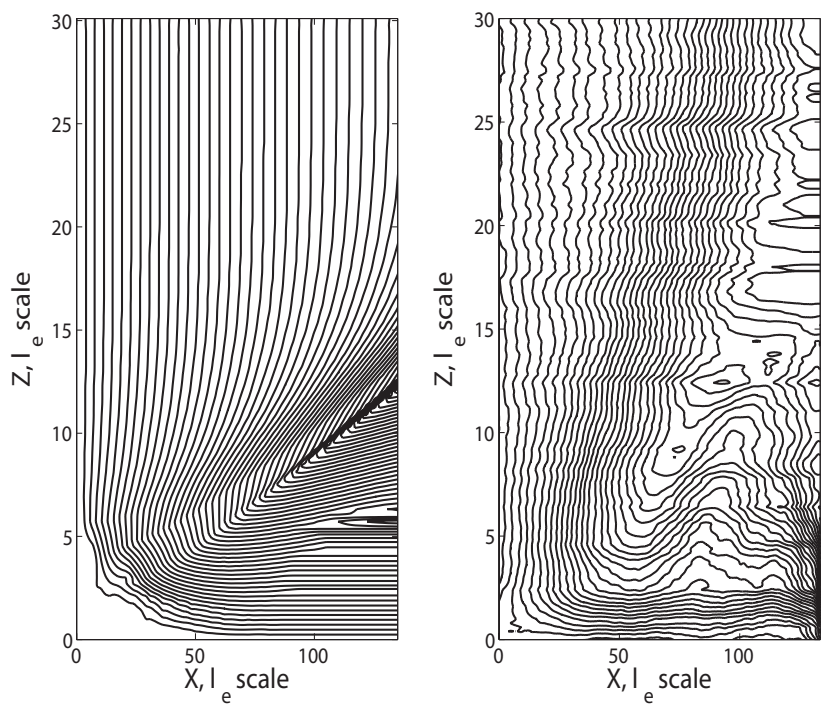

Fig. 3. Electron trajectories in $X Z$ plane: analytical model (on the left) and PIC simulation (on the right).

assumption is $j_{e} \gg j_{p}$, we restricted the region of the analytical model by the value $x=4$, where $j_{e} / j_{p} \approx 5$. Analogously, the upper boundary of the modelling region is restricted by the value $z_{\max }=30 l_{e}$. This value corresponds to $z_{\max }=4$ in the PIC simulation $\left(m_{p} / m_{e}=64\right)$ and $z_{\max }=0.7$ in the analytical modelling $\left(m_{p} / m_{e} \approx 1840\right)$. At last, the EDR half-length reaches $2 l_{p}$.

The PIC simulation provides us $d G / d A \equiv V_{e y}(A)$ and $B_{z}(x, 0)$ (see Fig. 2).

As for the total pressure $\Pi\left(x, z_{\max }\right)$, it turns out to be a linearly increasing but weakly varying quantity, $\Pi\left(0, z_{\max }\right)=0.61$ and $\Pi\left(4, z_{\max }\right)=0.66$. The last parameter of the analytical model is the reconnection rate $\epsilon \equiv E_{y}$. Its value obtained in the simulation is 0.2 .

The electron trajectories obtained from the analytical study and the PIC simulation are compared in Fig. 3. The magnetic field separatrix mapped by the electric current is clearly visible in both cases. In fact, this picture shows a classical Hall current structure (Sonnerup, 1979), observed in the magnetosphere (e.g. Alexeev et al., 2005) and in laboratory experiments (e.g. Cothran et al., 2005). The electron jet in $X$ direction is visible as well, in agreement with results of other authors (Daughton et al., 2006; Shay et al., 2007). The dependence of the velocity of this jet of $X$ is plotted in Fig. 4. The analytical model underestimates the electron velocity (approximately 50\%) as compared to that of the PIC simulation.

The proton velocities demonstrate a better agreement, with acceleration up to $1.5 \pm 0.1 V_{A}$ in both models. The proton trajectories and the magnetic field structure are presented in Fig. 5.
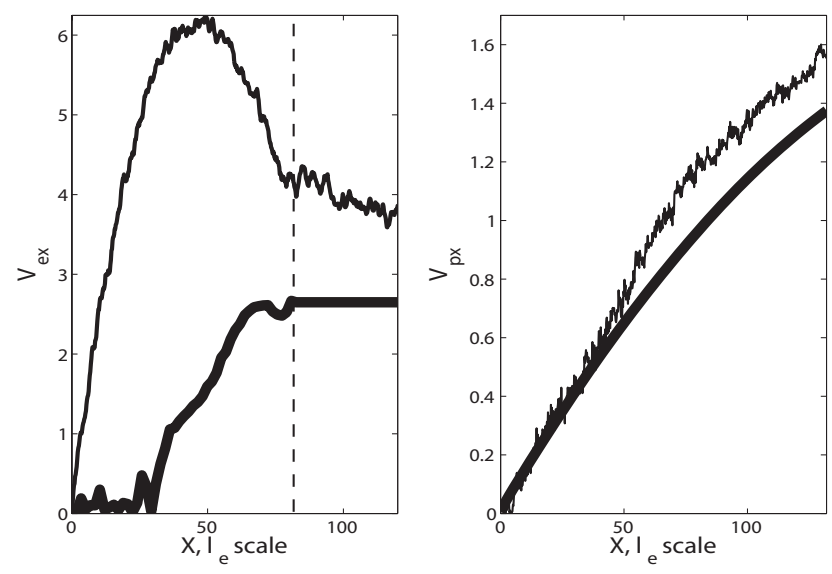

Fig. 4. Electron velocities $V_{e x}(x, 0)$ (left panel) and proton velocities $V_{p x}(x, 0)$ (right panel): analytical model (thick) and PIC simulation (thin). Vertical dished line on the left panel marks the EDR boundary. Strictly speaking, quantity $V_{e x}$ is undefined inside the EDR, $0<x<80$
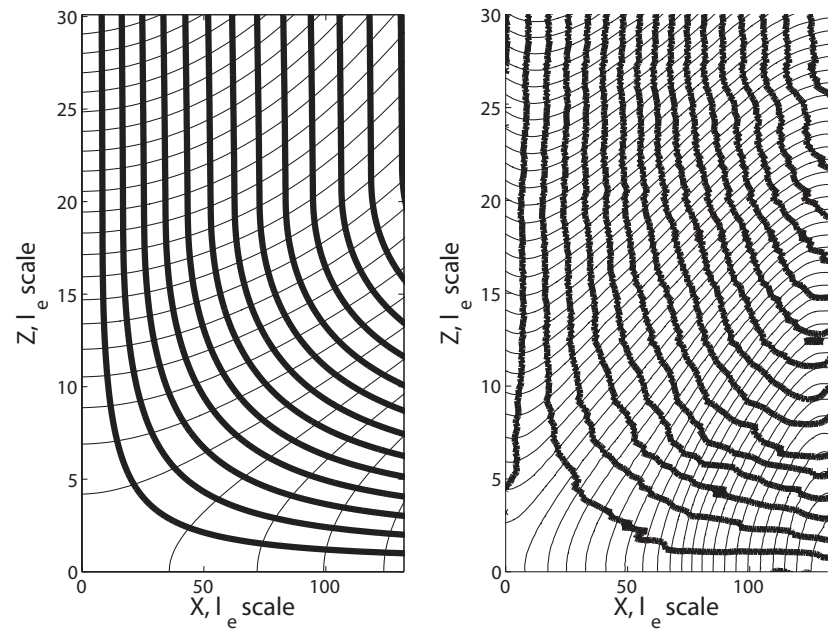

Fig. 5. Proton trajectories in $X Z$ plane (thick) and magnetic field lines (thin): analytical model (on the left) and PIC simulation (on the right).

At last, the electric fields $E_{z}$ shown in Fig. 6, are in good qualitative agreement as well. The localization of the extremum of $E_{z}$ corresponds to the jump of the electric potential across the separatrices as predicted.

\section{Conclusions}

One can see that the plasma characteristics obtained in both models are qualitatively equal. As for numeric values, the analytical model is not precise everywhere. While some values predicted are quite accurate (e.g., $\max \left|V_{e y}\right|, \max \left|V_{p}\right|$ ), 

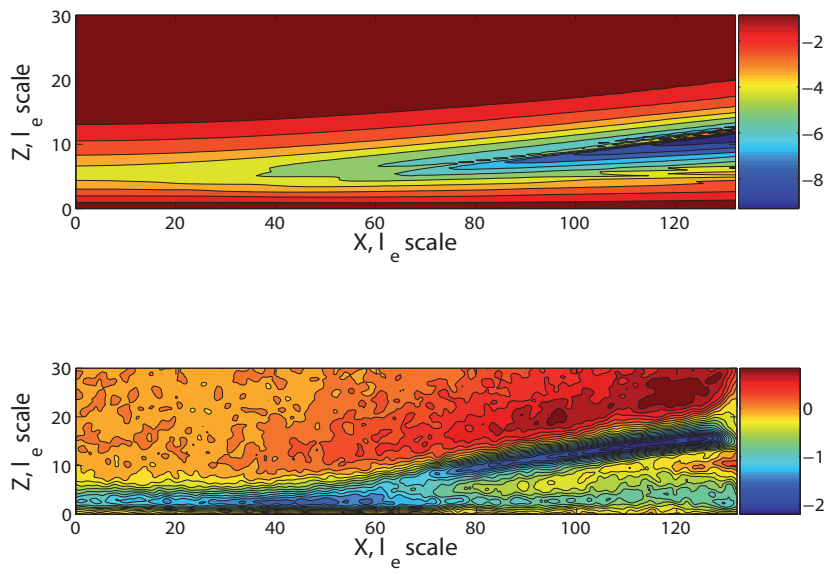

Fig. 6. Distribution of electric field $E_{z}$ in $X Z$ plane: analytical model (top) and PIC simulation (bottom).

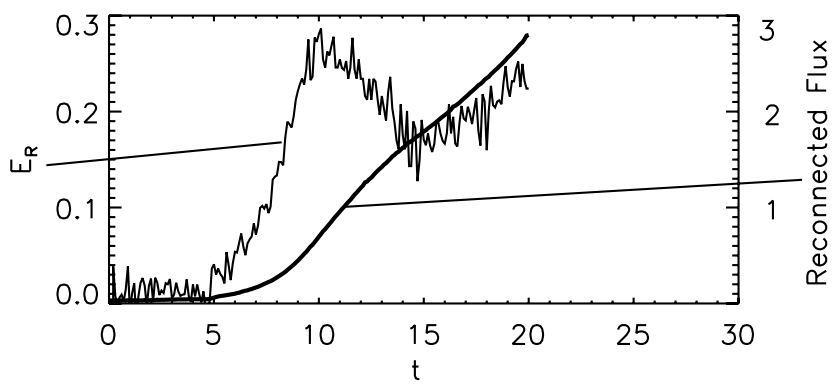

Fig. 7. PIC simulation: reconnected magnetic flux and reconnection electric field $E_{R}=E_{y}$ histories.

others differ noticeably (e.g., $V_{e x}, E_{z}$ ). We attribute this difference to the simplifications adopted. Namely, while outside the EDR $P_{e}$ is a weakly varying quantity, indeed, the situation is completely different inside the EDR. The electron pressure turns there to be an anisotropic tensor and $\nabla \hat{P}_{e}$ becomes the dominant term in the Ohm law. This term is responsible for the freezing-out of electrons in a thin and very stretched $\left(\sim 10 l_{p}\right)$ layer mapping the $X$ axis, called external EDR, where electron jets develop (see Fig. 6). Though this effect is completely out of the scope of our analytical study. Nevertheless, the analytical solution obtained demonstrates all essential Hall reconnection features and a close qualitative agreement with results of the PIC simulation.

Besides, this solution claims that a powerful mechanism of electron acceleration in the $X$-line direction is required. Accordingly to the estimation (26), it must accelerate electrons up to the electron Alfvén velocity inside the EDR and on the separatrices. At the downstream edge of the EDR, these accelerated electrons are deflected by the Lorentz force in $X$-direction and then get decelerated in the outflow region, pulling protons there.
Acknowledgements. This work is supported by RFBR, grant $\mathrm{N}$ 07-05-00776-a, and by Austrian "Fonds zur Förderung der wissenschaftlichen Forschung" under Project P20341-N16. The PIC simulations was performed by A. Divin at the University of Maryland, USA.

Topical Editor R. Nakamura thanks two anonymous referees for their help in evaluating this paper.

\section{References}

Alexeev, I. V., Owen, C. J., Fazakerley, A. N., Runov, A., Dewhurst, J. P., Balong, A., Rème, H., Klecker, B., and Kistler, L.: Cluster observations of currents in the plasma sheet during reconnection, Geophys. Res. Lett., 32, L03101, doi:10.1029/2004GL021420, 2005.

Bhattacharjee, A., Germaschewski, K., and Ng, C. S.: Current singularities: Drivers of impulsive reconnection, Phys. Plasmas, 12, 042305, doi:10.1063/1.1872893, 2005.

Birdsall, C. K. and Langdon. A. B.: Plasma Physics via Computer Simulation, in: Plasma Physics Series, edited by: Cowley, S., Stott, P., and Wilhelmsson, H., IOP Publ., Bristol, 1991.

Birn, J., Drake, J. F., Shay, M. A., Rogers, B. N., Denton, R. E., Hesse, M., and Kuznetsova, M., Ma, Z. W., Bhattacharjee, A., Otto, A., and Pritchett, P. L.: Geospace Environment Modeling (GEM) Magnetic Reconnection Challenge, J. Geophys. Res., 106, 3715-3719, 2001.

Biskamp, D.: Magnetic reconnection via current sheets, Phys. Fluids, 29, 1520-1531, 1986.

Biskamp D., Schwarz, E., and Drake, J. F.: Two-fluid theory of collisionless magnetic reconnection, Phys. Plasmas, 4, 1002-1009, 1997.

Biskamp, D.: Magnetic reconnection in Plasmas, Cambridge University Press, Cambridge, 2000.

Biskamp, D. and Schwarz, E.: Localization, the clue to the fast magnetic reconnection, Phys. Plasmas, 8(11), 4729-4731, 2001.

Büchner, J. and Daughton, W. S.: Role of current-alligned instabilities, in: RECONNECTION OF MAGNETIC FIELDS. Magnetohydrodynamics and Collisionless Theory and Observations, edited by: Birn, J. and Priest, E. R., Cambridge Univercity Press, Cambridge, pp. 144-153, 2007.

Coroniti, F. V.: Space plasma turbulent dissipation - reality or myth?, Space Sci. Rev., 42, 399-410, 1985.

Cothran, C. D., Landreman, M., Brown, M. R., and Matthaeus, W. H.: Generalized Ohm's law in a 3D reconnection experiment, Geophys. Res. Lett., 32(5), L03105, doi:10.1029/2005GL023973, 2005.

Daughton, W., Scudder, J., and Karimabadi, H.: Fully kinetic simulations of undriven magnetic reconnection with open boundary conditions, Phys. Plasmas, 13, 072101, doi:10.1063/1.2218817, 2006.

Divin, A. V., Sitnov, M. I., Swisdak, M., and Drake, J. F.: Reconnection onset in the magnetotail: Particle simulations with open boundary conditions, Geophys. Res. Lett., 34, L09109, doi:10.1029/2007GL029292, 2007.

Drake, J. F., Swisdak, M., Shay, M. A., Rogers, B. N., Zeiler, A., and Cattell, C.: Formation of electron holes and partical energization during magnetic reconnection, Science, 299, 873-877, 2003. 
Erkaev, N. V., Semenov, V. S., and Jamitzky, F.: Reconnection rate for the inhomogeneous resistivity Petschek model, Phys. Rev. Lett., 84(7), 1455-1455.8, 2000.

Erkaev, N. V., Semenov, V. S., and Biernat, H. K.: Two-dimensional MHD model of the reconnection diffusion region, Nonlin. Processes Geophys., 9, 131-138, 2002,

http://www.nonlin-processes-geophys.net/9/131/2002/.

Grasso, D., Porcelli, F., and Califano, F.: Hamiltonian magnetic reconnection, Plasma Phys. Contr. Fusion, 41, 1497-1515, 1999.

Hassam, A. B.: Collisional tearing in field-reversed configurations, Phys. Fluids, 27, 2877-2880, 1984.

Harris, E. G.: On a plasma sheath separating regions of oppositely directed magnetic field, Nuovo Cimento, 23, 115-121, 1962.

Hesse M., Schindler, K., Birn, J., and Kuznetsova, M.: The diffusion region in collisionless magnetic reconnection, Phys. Plasmas, 5, 1781-1786, 1999.

Huba, J. D., Gladd, N. T., and Paradopoulos, K.: The lower-hybriddrift instability as a source of anomalous resistivity for magnetic field reconnection, Geophys. Res. Lett., 4, 125-126, 1977.

Huba, J. D. and Rudakov, L. I.: Hall magnetic reconnection rate, Phys. Rev. Lett., 93, 175003-175003.8, 2004.

Kan, J. R.: Ion-wave current instabilities and anomalous resistivity, Phys. Fluids, 14, 2740-2747, 1971.

Korovinskiy, D. B., Semenov, V. S., Erkaev, N. V., Divin, A. V., and Biernat, H. K.: The 2.5-D analytical model of steady-state Hall magnetic reconnection, J. Geophys. Res., 113, A04205, doi:10.1029/2007JA012852, 2008.

Ma, Z. V. and Bhattacharjee, A.: Fast impulsive reconnection and current sheet intensification due to electron pressure gradients in semi-collisional plasmas, Geophys. Res. Lett., 23, 1673-1676, 1996.

Mandt, M. E., Denton, R. E., and Drake, J. F.: Transition to wistler mediated magnetic reconnection, Geophys. Res. Lett., 21, 7376, 1994.

Parker, E. N.: Sweet's mechanism for merging magnetic field in conducting fluids, J. Geophys. Res., 62, 509-520, 1957.

Petschek, H. E.: Magnetic field annihilation, in: AAS/NASA Symposium on the Physics of Solar Flares, edited by: Ness, W. N., 425, NASA, Washington, D.C., 1964.

Porcelli, F., Borgogno, D., Califano, F., Ottaviani, M., Grasso, D., and Pegoraro, F.: Recent advances in collisionless magnetic reconnection, Plasma Phys. Contr. Fusion, 44, B389-405, 2002.

Pritchett, P. L.: Geospace environmental modeling magnetic reconnection challenge: simulations with a full particle electromagnetic code, J. Geophys. Res., 106, 3783-3798, 2001.
Ricci, P., Lapenta, G., and Brackbill, J. U.: Gem recoonection challenge: Implicit kinetic simulations with the physical mass ratio, Geophys. Res. Lett., 29(23), 2088, doi:10.1029/2002GL015314, 2002.

Rogers, B. N., Denton, R. E., Drake, J. F., and Shay, M. A.: Role of dispersive waves in collisionless magnetic reconnection, Phys. Rev. Lett., 87(19), 195004-195004.4, 2001.

Sato, T. and Hayashi, T.: Externally driven magnetic reconnection and a powerfull magnetic energy converter, Phys. Fluids, 22, 1189-1202, 1979.

Schindler, K.: Physics of Space Plasma Activity, Cambridge University Press, Cambridge, 2007.

Shay, M. A. and Drake, J. F.: The role of electron dissipation on the rate of collisionless magnetic reconnection, Geophys. Res. Lett., 25, 3759-3762, 1998.

Shay, M. A., Drake, J. F., Denton, R. E., and Biskamp, D.: Structure of the dissipation region during collisionless magnetic reconnection, J. Geophys. Res., 103, 9165-9176, 1998.

Shay, M. A., Drake, J. F., Rogers, B. N., and Denton, R. E.: The scaling of collisionless magnetic reconnection for large systems, Geophys. Res. Lett., 26, 2163-2166, 1999.

Shay, M. A., Drake, J. F., Swisdak, M.: Two-scale structure of the electron dissipation region during collisionless magnetic reconnection, Phys. Rev. Lett., 99, 155002, doi:10.1103/PhysRevLett.99.155002, 2007.

Sonnerup, B. U. Ö.: Magnetic field reconnection, in: Solar System Plasma Physics, edited by: Lanzerotti, L. J., Kennel, C. F., and Parker, E. N., 3, 46, North Holland Pub., Amsterdam, 1979.

Sweet, P. A.: The neutral point theory of solar flares, in: Electromagnetic Phenomena in Cosmical Physics, edited by: Lehnert, B., Cambridge University Press, New York, 123, 1958.

Terasawa, T.: Hall current effect on tearing mode instability, Geophys. Res. Lett., 10, 475-478, 1983.

Uzdensky, D. A. and Kulsrud, R. M.: Physical origin of the quadrupole out-of-plane magnetic field in Hallmagnetohydrodynamics reconnection, Phys. Plasmas, 13, 062305, doi:10.1063/1.2209627, 2006.

Vasyliunas, V. M.: Theoretical models of magnetic field line merging, Rev. Geophys., 13, 303-336, 1975.

Wang, X., Bhattacharjee, A., and Ma, Z. W.: Scaling of collisionless forced reconnection, Phys. Rev. Lett., 87, 1-4, 2001.

Zeiler, A., Biskamp, D., Drake, J. F., Rogers, B. N., Shay, M. A., and Scholer, M.: Three-dimensional particle simulations of collisionless magnetic reconnection, J. Geophys. Res., 107(A9), 1230-1230.9, 2002. 